\title{
Bots, reality shows and Greek political parties: Tracking bots and their political propaganda in Greece
}

\author{
Ilias Stathatos \\ Mediterranean Institute for Investigative Reporting \\ istath@miir.gr \\ Dimitris Papaevagelou \\ Civic Information Office \\ dimitris.papaevagelou@andefined.com \\ Ioanna Louloudi \\ Mediterranean Institute for Investigative Reporting \\ jlou@miir.gr \\ Nikos Morfonios \\ Mediterranean Institute for Investigative Reporting \\ nmorf@miir.gr \\ Maria Sidiropoulou \\ Mediterranean Institute for Investigative Reporting \\ msid@miir.gr \\ Kostas Zafeiropoulos \\ Mediterranean Institute for Investigative Reporting \\ kzaf@miir.gr \\ Elvira Krithari \\ Mediterranean Institute for Investigative Reporting \\ ekrit@miir.gr
}

\begin{abstract}
The use of twitter bots in political campaigns and influence operations has come increasingly under the academic and journalistic limelight. In this study, we present the first data-driven journalistic investigation on the presence of bots on the Greek twittersphere that amplify political messaging of the main Greek political parties. We focused on two periods of high political and brand social media activity, the Thessaloniki International Fair (DETH) and the final of the reality show Greece's Next Top Model. Using an ensemble of algorithmic and visualization tools and classifiers, about 3.968 accounts were annotated on their political orientation and level of automation.
\end{abstract}

\section{Introduction}

The concept of propaganda is not a recent invention, but it is ever-present throughout history (Bazerman, 2016). However, during the last 10-15 years we have witnessed enormous changes: an ever-faster pace of news dissemination (John and Silberstein-Loeb, 2015), a massive volume of digital information, a growing mistrust in traditional media (Nicolaou and Giles, 2017), a worsening of journalists' work conditions (O'Donnell et al., 2016) and a spectacular rise of information on the Internet. According to a scientific report by the Joint Research Center of the European Commission (Martens et al., 2018), two out of three Internet users prefer to access the news through platforms based on algorithms, such as search engines, news aggregators and especially so- 
cial networks. Social networks enjoy a rapidly growing share in opinion making, especially in younger ages, in the economically active population and in urban centers.

One of the main conclusions reached after studying the dissemination of fake news worldwide during important events such as the 2016 US election, as well as the recent election in India -a country of one billion people- is this: the sources of a fake news story may vary, but the key for its successful dissemination is globally the same, and it is none other than automation.

Political campaigning has long incorporated predominance in the digital sphere as a strategy. The web offers political marketing a huge advantage: bigger and faster impact with smaller cost. In this sphere, automation without constant human presence succeeds in disseminating all kinds of messages by a relatively small number of individuals to a real mass audience with great speed (Woolley and Howard, 2017). And there is no better strategy than to combine automated processes with accounts of actual persons that have already formed a large crowd of followers. "How much of the Internet is fake?", was the question asked by "New York Magazine" (Read, 2018), in reference to studies according to which less than $60 \%$ of the total web traffic is human. Is automated political propaganda part of the Greek political discourse, and how large of a part it does it occupy? How does it function and what kind of political content is being disseminated?

These questions were the starting point for the Mediterranean Institute for Investigative Reporting (MIIR) team, consisting of six investigative, video and data journalists to attempt the first in-depth and data-based journalistic research about the fake and automated accounts on Twitter in Greece, in co-operation with the engineer Dimitris Papaevangelou of the Civic Information Office, who developed the algorithmic and visualization tools.

Our investigation tracked the process of opinion-making on social media, with a focus on Twitter, through the construction of virtual digital networks that aim at propaganda on behalf of politicians and political parties, as well as at the promotion of commercial TV productions. Based on quantitative and qual- itative data, we unveiled the ways in which Greek political parties, MPs, candidates and influencers, unknown and high-profile alike, rely on bots in order to promote their political positions, overwhelm their political adversaries and, first and foremost, influence those who set the political agenda.

\section{Previous Research}

The use of bots in social media is not a new phenomenon (Zeller Jr, 2006), but they have taken an oversized role in modern internet content consumption (Howard, 2018). In recent election battles they have an ever-increasing "disproportional" role (Shao et al., 2018) with impressive reach (Baraniuk, 2018).

Despite rigorous recent research (Shao et al., 2018) on the kind of content bots are spreading (Zaman) and their effects (Hjouji et al., 2018), we focused on the methodology one can detect a bot (Biersdorfer, 2017), as well as the patterns they follow (Hindman and Barash, 2018) in sharing their content (Confessore et al., 2018).

Moreover, we were interested in mapping the political clusters of Greek twitter in order to be able to conceptualize the role of bots and amplifiers in them. Finally, we were conscious to interrogate any particularities in the way bots were sharing their political messaging (Wojcik and Michael, 2018), and it will be evident later, this formed a big part of "successful" bots.

\section{Methodology - Data}

Our team examined only Twitter, as this is the most accessible and politicized social medium. We chose as a case study one of the most important pre-scheduled Greek political events of 2018, which we knew beforehand would generate an intense political controversy: the Thessaloniki International Fair in September 2018. This Fair was marked, among others, by much debate about the Prespa Agreement of June 2018 regarding the name of North Macedonia.

Based on software that was developed by Dimitris Papaevangelou, we drew all the tweets, retweets, replies, quotes and mentions from 7 September (before the Prime Minister's speech at the annual exhibition) to 17 September 2018 (after the opposition leader's speech), 
using specific keywords: \# $\Delta \mathrm{E} \Theta, \# \Delta \mathrm{E} \Theta 2018$,

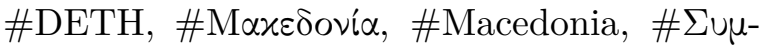

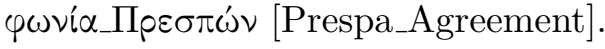

Using Twitter's Streaming API we collected about 173,000 separate actions from roughly 20,000 different accounts. We repeated the same procedure during the days of the final of the TV reality show Greece's Next Top Model (18-20 December 2018), where we drew about 8,200 accounts and 78,000 actions.

To collect the data from Twitter and for a particular set of hashtags and keywords, we used Twitter's Public Streaming API and not the Standard Search API, since we wanted to avoid the loss of important information such as deleted tweets. For that a custom command line interface was used -TwitterFarm, which uses Anaconda- and saved raw data information on an Elasticsearch Index online.

In order not to integrate into our database the same twitter actions multiple times, the collected actions were filtered according to their unique tweet id.

During the first step of analysis we extracted basic statistical information regarding the volume of tweets and type of tweet as it was very important to understand what was being discussed, when and how a message disseminated, which later helped us filter out specific time periods, tweet types and user accounts. To quick search and visualize statistics we used Kibana, Elasticsearch's data explorer.

The most important step during our analysis was to extract the network graph from raw data where we used "User" as nodes and tweet type (TWEET, RETWEET_OF, QUOTE_OF, REPLY_TO) as an edge.

We iterated over all data and extracted "User", "RetweetedStatus.User", "QuotedStatus.User" and performed "GetUserShow" requests for each "InReplyToUserID". Any parallel edges were ignored, since our purpose was to find relationships between users and not number of connections.

Next, after classifying the different accounts, we selected a random sample of 3,698 individual accounts from the repository of the tweets we had gathered. Four journalists of the Mediterranean Institute of Investigative Journalism analyzed, one by one, roughly 900 accounts each, reading at least 60 tweets from

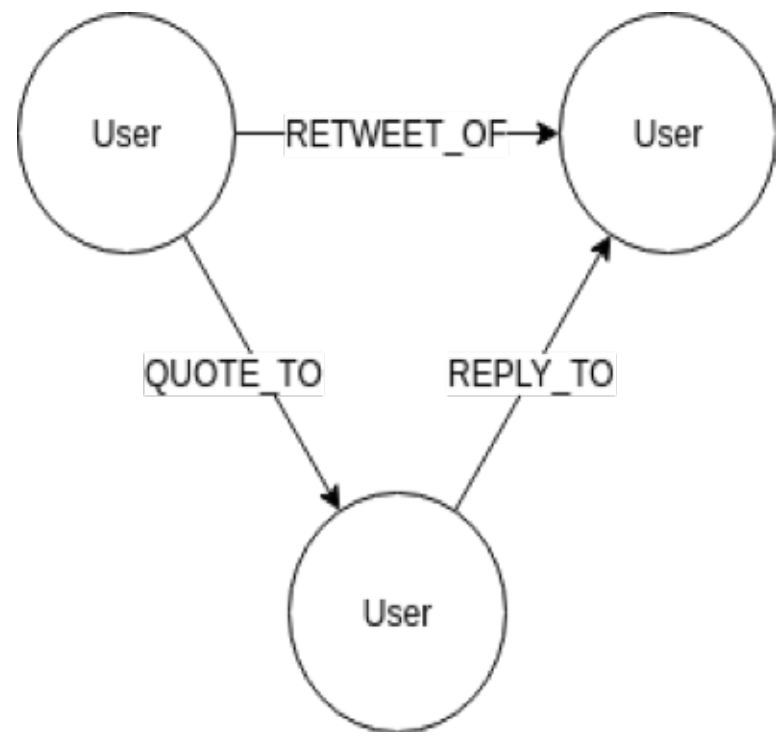

Figure 1

every account, and based on a series of homogenized, qualitative and quantitative criteria they categorized the accounts depending on their degree of automation and the type of user (unknown, active user, normal user, semi-automated, automated), as well as the user's estimated political orientation on a linear axis from Far Left to Far Right. In cases that we were unable to determine the degree of automation or the political orientation of an account based on its past activity, we categorized the account in question as "unknown". At the end of the procedure, we integrated the results in a unified database.

We created a graph to visualize all the clusters of the Greek accounts (both authentic and bots) of our sample. Every node in this graph represents a different Twitter account. Using algorithms, we searched for the forces that attract users to one another, thus forming a cluster, and also the forces that attract their clusters to one another (modularity).

The magnitude of each node (that is, of a Twitter account) depends on the degrees of interactions-interrelations of this account (edges) with all the other accounts within a cluster. In other words, the bigger the name shown on the graph, the larger the number of accounts that have interacted and reproduced its content. Correspondingly, the bigger a node, the larger the number of accounts it has reproduced, thus entering a cluster.

The clusters of Greek accounts on Twitter, 
both authentic and bots, depending on their mutual interaction and reproduction: In blue, the Nea Dimokratia cluster; in fuchsia, the SYRIZA cluster; in pink, the cluster of accounts talking about GNTM. In between (in light blue color), we find a whole array of accounts, from active users with varying political views to web influencers interconnected both with real accounts and with bots. In white is the cluster of far-right accounts.

The following graph represents the most influential nodes - namely, accounts, both authentic and bots, with the largest number of actions (tweets, retweets, quotes, mentions) on a daily basis.

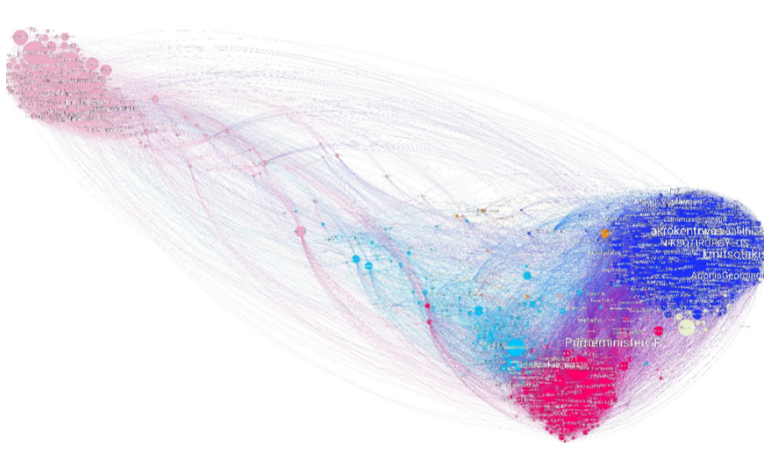

Figure 2

\section{Results}

One of our first findings was that many accounts were created shortly before the reference period of our research (7-17 September 2019) and they were shut down a few weeks later, either by the users themselves or by Twitter. $40.6 \%$ of the accounts we analyzed either had no political orientation or could not be categorized on a linear political axis from Far Left to Far Right and the Golden Dawn pro-Nazis. $8.2 \%$ of the remaining $59.4 \%$ expressed views clearly sympathetic to the communist Left, the extra-parliamentary Left and anarchism. Roughly $14.23 \%$ of the accounts expressed views close to the Center-Left, and $19.08 \%$ were closer to the liberal Center and the Center-Right.

Roughly $14.64 \%$ expressed views ranging from ultra-conservatism and right-wing populism to the nationalist and sectarian Far Right. Last but not least, $3.3 \%$ of the accounts were either openly supporters of Golden Dawn or sympathetic to Nazi and fascist ideas. These percentages are only circumstantial; they may be said to bring out an existing tendency, while also embodying the journalists' subjective judgments. They do not reflect in any case whatsoever a potential mapping of an election result, since the demographic characteristics of Twitter do not suffice to draw such conclusions.

\begin{tabular}{|l|c|c|}
\hline $\begin{array}{l}\text { DEGREE OF } \\
\text { AUTOMATION }\end{array}$ & $\begin{array}{l}\text { NUMBER OF } \\
\text { ACCOUNTS }\end{array}$ & PERCENTAGE \\
\hline Unknown & 409 & $11,06 \%$ \\
\hline Normal user & 1.339 & $36,21 \%$ \\
\hline Very active user & 1.474 & $39,86 \%$ \\
\hline $\begin{array}{l}\text { Semi-automated user } \\
\text { (Cyborg-bot) }\end{array}$ & 280 & $7,57 \%$ \\
\hline $\begin{array}{l}\text { Automated user } \\
\text { (Bot) }\end{array}$ & 196 & $5,30 \%$ \\
\hline TOTAL & 3.698 & $100,00 \%$ \\
\hline
\end{tabular}

Figure 3

The table above shows the degree of automation of the accounts we collected. In particular:

- We detected 476 bots out of a total of 3,698 accounts $(12.87 \%)$.

- The bots generated $57 \%$ of the total actions (tweets, retweets, mentions, quotes, replies, likes) during the reference period.

- We found bots that supported all the parties of the Greek parliament, except the Union of Centrists (Enosi Kentroon) party.

- SYRIZA and New Democracy are by far the political parties with the largest share of automated accounts. New Democracy declined to comment on our investigation. SYRIZA denied any connection with automated accounts. 
During the prime minister's speech in DETH, on 8 September 2018, the users' activity reaches its apex, with retweets owning the largest share. At the beginning of Alexis Tsipras' speech, on 9 pm, replies are by far exceeded by retweets, a feature often linked with increased automation (bots).

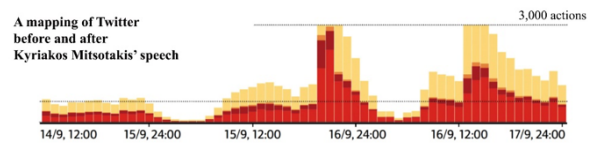

Figure 4

In accordance with Alexis Tsipras' speech, there is a sharp increase in the total number of actions during Kyriakos Mitsotakis' two-hour speech at the Vellidion Conference Center on 15 September 2018. Before Mitsotakis' speech, the number of posts on Twitter was smaller than the number of posts before the prime minister's speech.

- During both Alexis Tsipras' and Kyriakos Mitsotakis', the opposition leader, speeches in the $2018 \mathrm{DETH}$, including a period of a few hours before and after the speeches, there was a spectacular increase in the number of users' actions. There is a comparably sharp increase in bots' activity during the same time-period as well, obviously with a view to political propaganda.

- Many bots are interconnected and also used to promote TV productions.

- The important thing is not the absolute number of automated accounts, but the volume and the intensity of the digital noise they produce. A small number of bots is enough to influence public opinion (Zaman).

- We confirmed through a computational method what we had found through our journalistic analysis: that the two major political parties have the hegemony concerning the total content produced on the web, and they fight each other over it.

- We confirmed that the political parties, especially the two major ones, are significantly promoted by bots in obscure and illegitimate ways.
- We found out how particular actual persons (named or anonymous users) are connected with automated accounts.

- We detected 6 major clusters, represented on the graph. We gave each one of the larger clusters a different name. In between the three main clusters (the blue one, New Democracy; the fuchsia one, SYRIZA; the pink one, GNTM), there are the light blue users (nodes), which show high activity but do not belong to any of the three main clusters. As mentioned above, this is an in-between space where one can find a whole array of accounts, from active users with varying political views to web influencers interconnected both with real accounts and with bots. As we already said, the white color next to the cluster of New Democracy represents the cluster of accounts that express nationalist and far-right opinions. The orange color represents the bots that show a generic behavior with standard cues or mutual salutations (good morning, goodnight), in an attempt to pass as real persons.

It is noteworthy that after we constructed the graph that interconnects the accounts, we observed that in the surrounding area or very close to the clusters of New Democracy or SYRIZA there are a few accounts belonging to users that are known not to be supporters of the corresponding political party. This happens because there is a regular interaction among these users (e.g., journalists who reproduce politicians' tweets or accounts that receive many aggressive comments). This is why Alexis Tsipras' official account, @PrimeministerGR, is not located at the center of the SYRIZA cluster but in the space between SYRIZA and Nea Dimokratia, since it is obviously reproduced and commented by everyone.

- Proximity to one of the major clusters does not necessarily mean political affiliation.

- The far-right cluster (the white one) is located much closer to, and interacts more frequently with, the New Democracy cluster, the center-right official opposition party. 


\section{The use of brands and lifestyle content for wider political messaging dissemination}

The account P. P. is a typical case of a camouflaged automated account that serves a double purpose: targeted and anonymous political propaganda along with commercial promotion. This account with the very common Greek name, with a gull as its profile photo and a horse as its general photo, had as of 30 March 2019 46,500 followers, while it followed itself 48,600 other users.

In the reference period of our research, this user seemed to perform about 502 actions per day on Twitter. Common sense along with the relevant literature agree that it is very rare for a human to perform so many actions within a day. A highly active user usually does not perform more than 40-50 actions per day, according to DFRlab's guidelines.

$\mathrm{P}$ 's followers are literally from all over the world; they speak different languages and, naturally, many of them are also fake or automated account. One eminent Greek politician that follow this account is New Democracy MP and former minister Nikos Dendias.

It constantly reproduces tweets by other non-political accounts, adding various and mostly non-political comments of its own, allegedly "profound" dictums, pictures of top models or famous soccer players such as Lionel Messi, images of landscapes, flowers, etc

The account with the telling name "Hellenic Fighter" retweeted here is also automated. One needs only look at its quantitative data. On the "Fighter's" profile picture, we see a Byzantine eagle, a warrior, the Greek flag, Acropolis and Hagia Sophia in Istanbul. The "Fighter's" motto: "My home... Greece!!! My religion... Christian Orthodox!!! Greece, thank you for having given birth to me!!!". This nationalist account is followed by the official accounts of Nikos Dendias and Dora Bakogianni, both prominent politicians of the New Democracy Party.

Suddenly, amidst hundreds of irrelevant comments and photos made specifically to draw the attention of thousands of users on Twitter, we see a tweet by New Democracy MP Dimitris Stamatis, who, among other things, is hitting hard at Alexis Tsipras and
Stavros Theodorakis for "selling off" Macedonia.

Two days later, on 27 March 2019, the same account reproduces a tweet, promoting Stelios Kymbouropoulos' candidacy for the European Parliament with New Democracy and at the same time calling "good-for-nothing bullies" candidates Rallia Christidou, Lydia Koniordou and Petros Kokkalis, all supported by SYRIZA.

During DETH, "Hellenic Fighter", along with its nationalist slogans and comments, seizes the opportunity to question the republican constitution itself, writing that "in this country, the Republic has failed us". The afore-mentioned case is crucial to our investigation because what we have here is an automated account (Hellenic Fighter), which is connected with other clusters of automated accounts (botnets) that, besides reproducing one another, connect to and reproduce the content of an account that clearly is not fake nor automated: the account "Lavrentis Beria". Anyone who has even a remote knowledge of the Greek political Twitter these last years is surely familiar with this account, which is a typical case of a "celebrity account" with a pseudonym and a large crowd of followers.

Looking at the profile of the account Paristzina @paristzina, we read its selfpresentation as "single, NUTS ANIMAL LOVER VOLUNTEER AT MUNICIPAL ANIMAL WELFARE ASSOCIATION", born somewhere in San Francisco, California. The content reproduced by the account confirms the "animal-loving" part, since it often retweets popular Greek and foreign accounts promoting animal welfare. It also retweets pictures and videos with happy and cute pets posted by other users on Twitter.

Besides cute pets, "Paris Tzina" retweets photos of beautiful landscapes, family moments, couples hugging and kissing, and halfnaked women, all generated by other automated accounts. In reality, this is a botnet, in which the account Aquarius @tzinaforever, created in January 2018, has 36,600 tweets, 126,000 likes, 9,194 followers and 7,149 followed, posts on a regular basis pictures of halfnaked women to draw other users' attention, while also retweeting standard salutations and 
generic cues from other accounts.

Similar cases include user "Lena" @lena_lenaki_len, which, according to our data, tweets 1,131 times per day; user "Helen Angel" @AngelAngelopou1, with 935 tweets per day; and user "Marilena" et_pd4, with 352 actions per day and a female profile photo that is easily detectable on Pinterest, the popular online free-access photo stock. All these robots pretend to talk to one another and exchange greetings - a typical indicator or bot-related automated activity.

In the same context, we singled out the account "Anna" @Anast1257, with which "Paris Tzina" has conversations with the participation - surprise, surprise - of our old friend "Hellenic Fighter" @kostas3012, thus highlighting the complex yet tight structure of a botnet. While showing pictures of cute pets and exchanging profound philosophical verses, some interaction with accounts that spread nationalist hatred seems quite in order.

But the mission given to "Paris Tzina" by its maker is not yet accomplished; the account has also to make some pro-New Democracy political propaganda, retweeting, amidst landscapes and pets, posts by MPs such as D. Stamatis, "liking" Maria Spyraki and Adonis Georgiadis, and regularly reproducing accounts known for their support to New Democracy, such as Georgy Zhukov @GeorgyZhukov, Lavrentis Beria @LavrentisBeria, Petaloudomachos Patra @hatz_patty, Anachoritis PSOFOS @MakeMyDayPunk and George Lee @Harhalas. A special mention is in order for the regular reproduction of Nikos Sotiropoulos, a known account that supports New Democracy, owned by a partner of the Patras Commercial Association, a publicist specialized in social media. Our investigation has shown that Sotiropoulos is very active on Twitter, a major influencer that usually makes posts against SYRIZA and has also a wide network of interconnected accounts.

That "Paris Tzina" is in fact affiliated with New Democracy is further confirmed by data during DETH, when this account, with an average of 415 actions per day, tweeted regarding DETH a total of 283 times for our reference period, mostly retweeting content against Tsipras and SYRIZA while wishing tirelessly good morning and goodnight to the world.

While examining the accounts with the most actions per day during DETH, we encountered an account named "Xenophon Vasilopoulos" with user name b8QTRcDJw2hMd4O - an obvious product of a generator of alphanumerics. The account tweeted about 715 times per day.

On its profile, one cannot help but notice a huge Greek flag with the Macedonian sun and the shadow of a warrior with a sword on the background; the profile picture is an ancient Greek helmet with the famous phrase Molon labe, "Come and take (them)".

This is an automated far-right account directly connected with the official website of Golden Dawn and reproducing all the articles posted by the neo-Nazi party. In just 17 months since its creation, the account has made 193,000 tweets and 130,000 likes, following 5,000 accounts and followed by 1,818.

The account in question regularly reproduces the content of the personal accounts of Golden Dawn leader Nikos Michaloliakos and Golden Dawn MPs such as E. Zaroulia, I. Kasidiaris, Ch. Pappas, etc.

It is noteworthy that the account in question also retweets its own tweets in the few original comments it posts, all nationalistic ones. There are also several attacks against politicians, the apex of which was the days of Tsipras' visit to North Macedonia in early April. During those days, the account went wild, posting insults and threats against the Greek and Macedonian ministers of Foreign Affairs, Nikola Dimitrov and Georgios Katrougalos, after their joint press conference.

The following image shows in white the cluster of Greek far-right accounts. Amidst them all, circled in red, one may see the Golden Dawn bot, "Xenophon", next to Ilias Kasidiaris' and Christos Pappas' accounts.

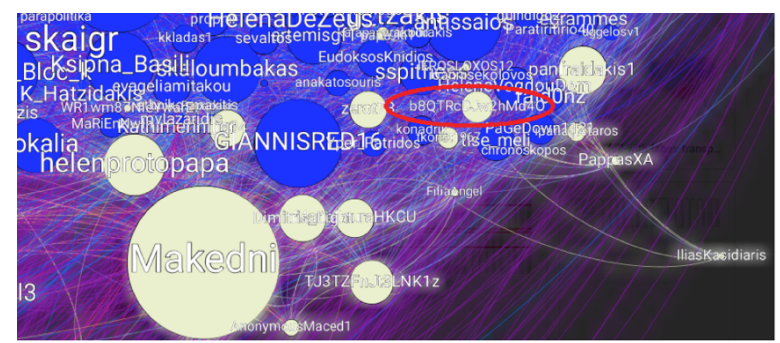

Figure 5 


\section{Brands}

During our research in the 3,698 accounts that tweeted about DETH, we observed that there were many users that used to tweet persistently about TV reality shows, quiz shows, and lifestyle shows. However, amidst posts by such users, we also detected a regular and unedited reproduction of political discourse, mostly by MPs and members or the two largest Greek parties, as well as by accounts known to support them on Twitter.

On that basis, we formulated the following question: Which of the 20,000 accounts that tweeted about Deth2018 also commented about the final of GNTM in December 2018?

After collecting and collating the relevant data, we saw that, out of a total of 8,200 accounts that tweeted about the GNTM final, 1,838 users had also tweeted about DETH from 7 to 17 September 2018. A benevolent observer might conclude that this behavior is more or less normal on social media. But is this actually true? In fact, amidst these 1,838 accounts, we detected some very interesting cases. After a quantitative and qualitative analysis of their posts, we found out that these cases showed an automated behavior in transmitting two seemingly different messages: the promotion of TV shows and the promotion of the political discourse of the two parties.

A quite telling case is the account with the peculiar name "Modest Hunk". ts quantitative features (20 tweets per day) do not cry out automation, a careful analysis of its content proves otherwise. he user retweets accounts with content relevant to almost all the reality and quiz shows of the Greek TV. And while the content of the above seems to belong to a user totally into this kind of TV shows, all of a sudden the account intermittently retweets the accounts of prime minister Alexis Tsipras, of SYRIZA ministers and MPs such as N. Pappas, D. Papadimoulis, E. Achtsioglou, the SYRIZA-affiliated "Memory Team", as well as various accounts that openly support SYRIZA. Another striking finding is that during DETH, "Modest Hunk" proved to be particularly active, performing a total of 136 actions, as opposed to 162 during the GNTM final.

\section{Conclusion}

This investigation is a synergy between computational tools and journalistic interrogation that we strongly believe is needed in order to address the pressing issue of truth and trust online. We confirmed through a computational method what we had found through our journalistic analysis: that the two major political parties have the hegemony concerning the total content produced on the web, and they fight each other over it. We further confirmed that the political parties, especially the two major ones, are significantly promoted by bots in obscure and illegitimate ways. We found out how particular actual persons (named or anonymous users) are connected with automated accounts.

Moreover, we detected the sharing of lifestyle and brand-related content by bot accounts who also shared political content, in accordance with recent research, which forced us to conceptualize them not just as quantitative amplifiers of a political message but as social actors incorporating tropes and affectations of the culture into which they operate.

According to sociologist Manuel Castells, media in themselves do not have the power to change things but are by and large the "field" where the ones with the power to move things are determined The same seems to be true for social media, this new arena of political propaganda. If anything, bots are surely there to influence people. Fifty years ago, philosopher Hannah Arendt (Arendt, 1973) wrote: "The result of a consistent and total substitution of lies for factual truth is not that the lies will now be accepted as truth, and truth be defamed as lies, but that the sense by which we take our bearings in the real world - and the category of truth vs. falsehood is among the mental means to this end - is being destroyed" . In the end, the goal may perhaps not be to determine a political agenda and a domain of public issues based upon fake news, but to establish an agenda in public life that will not separate truth and falsehood. In the end, what is currently at risk may not be truth, but trust. 


\section{References}

H. Arendt. 1973. The origins of totalitarianism. Houghton Mifflin Harcourt.

C. Baraniuk. 2018. How twitter bots help fuel political feuds. Scientific American.

C. Bazerman. 2016. Propaganda and rhetoric in democracy: history, theory, analysis. SIU Press.

J. D. Biersdorfer. 2017. Twitter bot — or not? The New York Times.

N. Confessore, G. JX Dance, R. Harris, and M. Hansen. 2018. The follower factory. The New York Times, 27.

M. Hindman and V. Barash. 2018. Disinformation, 'fake news' and influence campaigns on twitter. Knight Foundation.

Z. el Hjouji, D. S. Hunter, N. G. d. Mesnards, and T. Zaman. 2018. The impact of bots on opinions in social networks. arXiv preprint arXiv:1810.12398.

P. N. Howard. 2018. How political campaigns weaponize social media bots. IEEE Spectrum: Technology, Engineering, and Science News.

R.R. John and J. Silberstein-Loeb. 2015. Making news: The political economy of journalism in Britain and America from the glorious revolution to the internet. Oxford University Press, USA.

B. Martens, L. Aguiar, E. Gomez-Herrera, and F. Mueller-Langer. 2018. The digital transformation of news media and the rise of disinformation and fake news.

A. Nicolaou and C. Giles. 2017. Public trust in media at all time low, research shows. Financial Times, 16.

P. O'Donnell, L. Zion, and M. Sherwood. 2016. Where do journalists go after newsroom job cuts? Journalism Practice, 10(1):35-51.

M. Read. 2018. How much of the internet is fake. Turns Out, a Lot of It, Actually. New York Magazine (Intelligencer), December, 26.

C. Shao, Varol O. Yang K-C. Flammini A. Ciampaglia, G. L., and F. Menczer. 2018. The spread of low-credibility content by social bots. Nature communications, 9(1):4787.

S. Wojcik and B. Michael. 2018. The news that bots share on twitter usually isn't political. Pew Research Center.

S.C. Woolley and P.N. Howard. 2017. Computational propaganda worldwide: Executive summary. Computational Propaganda Research Project, pages 2017-11.
T. Zaman. Even a few bots can shift public opinion in big ways. The Conversation.

T. Zeller Jr. 2006. Gaming the search engine, in a political season. New York Times, November, 6. 